\title{
Fluorescent-Image Guidance in Robotic Subtotal Gastrectomy
}

\author{
Naruhiko Ikoma, MD, MS, Brian D. Badgwell, MD, MS, and Paul Mansfield, MD \\ Department of Surgical Oncology, The University of Texas MD Anderson Cancer Center, Houston, TX
}

\begin{abstract}
Robotic surgery technology has significant advantages, but its limitations include lack of tactile feedback. Fluorescent-imaging technology, part of the da Vinci robotic surgery system, helps to overcome this lack of feedback and improve safety. This video demonstrates the utility of fluorescent-image guidance in robotic subtotal gastrectomy. First, peritumoral injection of indocyanine green helps localize the primary lesion. Second, it enables visualization of sentinel lymphatic flow to guide the extent of lymph node dissection. Third, intravenous injection of indocyanine green confirms adequate tissue perfusion of the remnant stomach. Lastly, fluorescent angiography also visualizes the jejunal arterial arcade in the mesentery. In summary, we consider that fluorescent-image guidance may provide important intraoperative information that
\end{abstract}

supports techniques of robotic subtotal gastrectomy. Further studies to validate the benefits of fluorescent-image guidance are needed.

FUNDING Supported in part by the US National Institutes of Health under Cancer Center Support Grant P30CA016672; the Clinical Trials Support Resource was used.

DISCLOSURE The authors have no conflicts of interest to disclose.

Publisher's Note Springer Nature remains neutral with regard to jurisdictional claims in published maps and institutional affiliations.

This manuscript was selected for oral presentation at the Society of Surgical Oncology Annual Cancer Symposium (28 March 2020, Boston, MA).

Electronic supplementary material The online version of this article (https://doi.org/10.1245/s10434-020-08523-5) contains supplementary material, which is available to authorized users.

(C) Society of Surgical Oncology 2020

First Received: 22 January 2020;

Published Online: 7 May 2020

N. Ikoma, MD, MS

e-mail: nikoma@mdanderson.org 